\title{
Aesthetic Experience of Field Cognitive Style in the Appreciation of Cursive and Running Scripts: An Eye Movement Study
}

\author{
Hui Zhang, Ping Wang, Tinghu Kang* \\ School of Psychology, Northwest Normal University, Lanzhou, China \\ Email: *kangyan313@126.com
}

How to cite this paper: Zhang, H., Wang, P., \& Kang, T. H. (2020). Aesthetic Experience of Field Cognitive Style in the Appreciation of Cursive and Running Scripts: An Eye Movement Study. Art and Design Review, 8, 215-227.

https://doi.org/10.4236/adr.2020.84017

Received: September 20, 2020

Accepted: November 14, 2020

Published: November 17, 2020

Copyright $\odot 2020$ by author(s) and Scientific Research Publishing Inc. This work is licensed under the Creative Commons Attribution International License (CC BY 4.0).

http://creativecommons.org/licenses/by/4.0/

\begin{abstract}
This study compares the characteristics of the aesthetic experience of different cognitive styles in calligraphy style. The study used a cursive script and running script as experimental materials and the EyeLink 1000 Plus eye tracker to record eye movements while viewing calligraphy. The results showed that, in the overall analysis, there were differences in the field cognition style in total fixation counts, saccade amplitude, and saccade counts and differences in the calligraphic style in total fixation counts and saccade counts. Further local analysis found significant differences in the field cognitive style in mean pupil diameter, fixation counts, and regression in count, and that there were differences in fixation counts and regression in count in the calligraphic style, as well as interactions with the area of interest. The results indicate that the field cognitive style is characterized by different aesthetic experiences in calligraphy appreciation and that there are aesthetic preferences in calligraphy style.
\end{abstract}

\section{Keywords}

Cognitive Styles, Cursive Script, Running Script, Aesthetic Preferences

\section{Introduction}

Psychological aesthetics is an interesting field. The central question in this field is what evokes the aesthetic experience in a work of art and what factors influence aesthetic preferences (Leder, Belke, Oeberst, \& Augustin, 2004). In Western culture, the knowledge and experience of visual art mainly come from post-impressionist painting (Boselie \& Leeuwenberg, 1985), realistic painting (Yarbus, 1967), classical painting, and cubist art works (Buswell, 1935; Kapoula, 
Daunys, Herbez, \& Yang, 2009). In Oriental culture, calligraphy is one of the important fields of visual aesthetics research.

\section{Literature Review}

Chinese calligraphy, the art of Chinese characters written with a soft brush, is widely regarded as a unique form of art (Kao et al., 2014; Kao, 2010; Chu, Huang, \& Ouyang, 2018). Calligraphy originated early and has various forms. Common forms of calligraphy include cursive script, running script, regular script, seal script, and official script. Cursive calligraphy is a special form of calligraphy involving the cursive writing of Chinese characters. The difference between cursive script and other calligraphy works lies in the uncertainty of its writing style and the changes in the internal structure of strokes caused by the speed of writing. The diversity of uncertainty and changes in creation make cursive script comprehensible (Yu \& Peng, 2005); that is, it is difficult for nonprofessional practitioners to recognize its content. A cursive script is characterized by not only its smooth flow, but also the independent and neat beauty of the characters.

The study found that calligraphy style was related to the person's cognition and aesthetic appreciation and could even reflect the artist's personality (Zhuang, Lu, \& Wu, 2009). For artists, the purpose of creation is not only to provide the viewer with an opportunity to recognize the work, but also to awaken the viewer's subjective response to the style and structure of the work to gain an experience of "beauty" (Cupchik, Vartanian, Crawley, \& Mikulis, 2009). Cupchik categorizes the aesthetic process of the aesthetic subject of artistic works into two categories: cognition-based aesthetics and emotion-based aesthetics. The surface reaction process of cognition-based aesthetics consists of three stages: perception, cognition, and reflection. In the perception stage, the aesthetic subject perceives the basic qualities of the work with the senses. In the cognition stage, the aesthetic subject identifies the style, color, and subject matter of the work. In the reflection stage, the aesthetic subject compares, judges, and appreciates the work. The deep response process of cognition-based aesthetics consists of three stages: symbolic, projective, and transcendental. In the symbolic stage, the aesthetic subject's conscious preferences and experiences influence its outward responses. In the projective stage, the aesthetic subject's unconscious control influences its aesthetic responses. In the transcendental stage, the aesthetic subject's judgment of the work is sublimated, and the aesthetic subject will further perceive the self and life by experiencing the meaning of the work. The superficial reaction of emotion-based aesthetics results when the aesthetic subject finds the work interesting or has a certain emotion for the work from her own needs. The deep reaction of emotion-based aesthetics occurs when the aesthetic subject prefers a certain work because of her own personality traits or preferences. Aesthetic preference is an individual's aesthetic psychological tendency to give priority in attention to or to examine a certain type of aesthetic object or a certain form, style, or subject matter of artwork. To some extent, it 
determines the directionality and selectivity of an individual's aesthetic activities, and it reflects an individual's emotionally charged experience and appreciation of aesthetic objects. Therefore, whether aesthetic preferences exist within the art field during the aesthetic experience of calligraphic style will be the key concern of this study.

Related research has found that there are individual differences in aesthetic preferences within the visual arts due to such factors as gender (Cupchik \& Gebotys, 1990), level of expertise (Augustin \& Leder, 2006; Hekkert \& Van Wieringen, 1996), the amount of training in arts that an individual has received, and so on (Boselie \& Leeuwenberg, 1985; Winston \& Cupchik, 1992; Vogt \& Magnussen, 2007). In the late twentieth century, Witkin suggested that cognitive style affects aesthetic preferences (Witkin, Goodenough, \& Oltman, 1979). Cognitive style usually refers to the characteristic ways in which individuals perceive environmental stimuli, organizations, and cued information (Messick, 1984; Tennant, 1988). Accordingly, Witkin proposed the field cognition style and divided it into field dependence (FD) and field independence (FDI). Field-independent individuals have difficulty separating incoming information from their surroundings, are more susceptible to external cues, and are not selective in their information intake. Field-dependent individuals can separate the most basic information from its context relatively easily, are more susceptible to internal than external cues, and are selective in their information intake (Riding \& Cheema, 1991; Zhang, 2004; Witkin, Goodenough, \& Oltman, 1979; Guisande, Páramo, Tinajero, \& Almeida, 2007). Zhang (2004) argued that visual perception studies highlight a fundamental difference between the two. That is, the overall visual structure of the stimulus material affects field-dependent pattern recognition, whereas the field-independent type is more likely to decompose the visual structure of complex images and discern their unique parts.

This study attempts to analyze the aesthetic characteristics of the field cognitive style in cursive and running scripts. A detailed cognitive analysis of field-independent and field-dependent subjects' eye-movement indicators when viewing cursive and running script works was conducted using eye movement experiments. Eye-tracking technology has been used by researchers as a tool to reveal the perceptual and cognitive processes hidden behind the perception and aesthetic evaluation of artworks (Nodine \& Krupinski, 2003), and its emergence and popularity have opened up new possibilities for conducting quantitative studies of visual information processing (Dong et al., 2019). Attention studies have shown that the eye movement method is a reliable indicator of significant selection (Rizzolatti, Riggio, Dascola, \& Umilta, 1987). For example, fixation-like eye movement indicators can reflect the extent to which they cognitively process visual material, and pupil diameter measurements have been used as reliable unconscious physiological indicators of emotional arousal (Bradley et al., 2010). Visual arts research has also identified the potential of eye-tracking techniques to reveal the attentional and cognitive processes that occur during aesthetic events and the aesthetic evaluation of artworks (Locher, 2006). 


\section{Method}

Participants. The test is based on three sets of 9 simple and 20 complex figures from the Mosaic Figure Test (EFT), revised by the Department of Psychology, Beijing Normal University, and it has a reliability coefficient of 0.90 . The test is based on the cognitive style tendencies of the participants. The test material is pre-predicted and standardized, and the test taker is asked to identify a simple pattern given prior to a complex pattern. The quiz duration was five minutes. A final score greater than 50 indicates a preference for the field-independent type, while a score less than 50 indicates a preference for the field-dependent type. Statistical table of the subjects is shown in Table 1, all of whom had normal or corrected visual acuity and had never participated in a similar experiment before. The subjects were given souvenirs after the experiment. All subjects signed informed consent. The study was approved by the Ethics Committee of the School of Psychology, Northwest Normal University.

Table 1. Statistical table of the subjects.

\begin{tabular}{ccc}
\hline & frequency & percentage \\
\hline Field cognition style & 21 & \\
field-dependence & 19 & $52.5 \%$ \\
field-independent & & $47.5 \%$ \\
Gender & 14 & \\
male & 26 & $35 \%$ \\
female & & $65 \%$ \\
\hline
\end{tabular}

Stimuli. The experimental materials included the following two kinds: 1) cursive calligraphy post: eight pictures of calligraphic scrolls, including four each by Zhang $\mathrm{Xu}$ and $\mathrm{Mi}$ Fei in cursive (Figure 1);2) running script: selections from Wang Xizhi's "Lantingji Preface" (known as "the world's first running script", Figure 2) and Zhao Mengfu's "Luo Shenfu" in the complete version, according

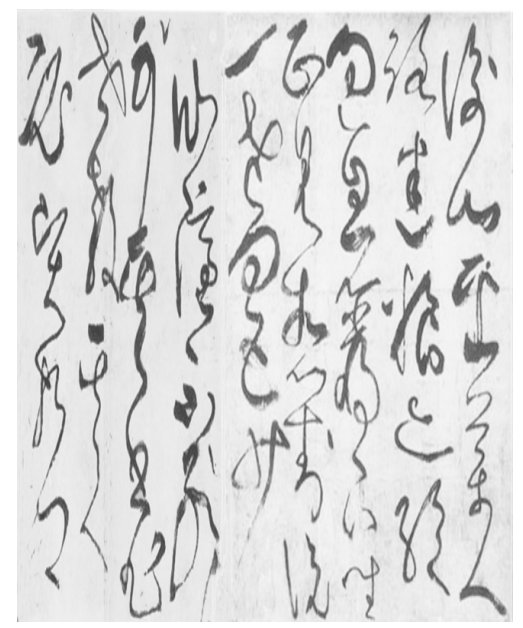

Figure 1. Cursive script. 


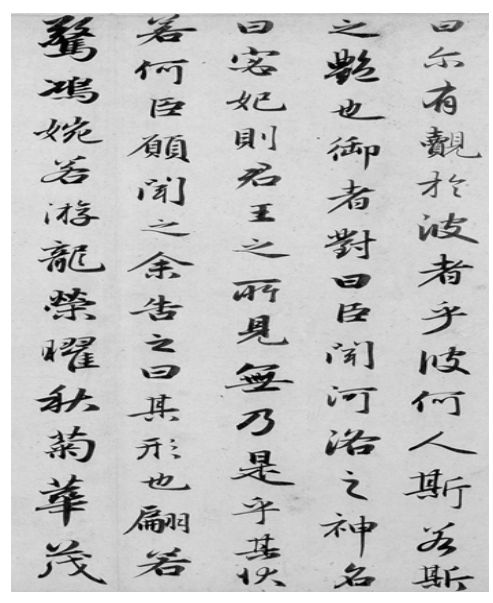

Figure 2. Running script.

to the content of the uniformly cut 20 pictures of material. All word images were edited using Adobe Photoshop CS5 to meet the experimental requirements of the experimental material and were edited as black and white images with 50\% brightness and a size of $1024 \times 566$ pixels.

Apparatus. The Eye Link 1000 Plus (SR Research, Canada) Desktop eye tracking device, $1000 \mathrm{~Hz}$ sampling rate, 19-inch display (1024 × 768 resolution), monocular pupil-corneal reflection recording, 9-point calibration, chin and Forehead Rest to fix the distance between the eyes and the stimulus screen (60 $\mathrm{cm}$ ). The visual field of the stimulus material has a horizontal viewing angle of 33.9 degrees and a vertical viewing angle of 37.3 degrees.

Procedures. The experiment was divided into three phases. In Phase 1, the participant was taken to the office and asked to fill out a registration form with basic information (e.g., gender and age) and was given five minutes to complete the three sets of questions for the EFT. In Phase 2, the participant was brought to the eye tracking lab and was seated $65 \mathrm{~cm}$ from the computer screen with their chin and forehead fixed. After calibration, the subjects were first presented with instructions. The participant was informed that the experiment would begin with a red " + " gaze point, and then a picture of a calligraphic writing tablet would be presented on the computer screen in full screen for the participant to enjoy freely. After each picture, the participant was asked to answer questions about the calligraphy (e.g., whether the calligraphy was smooth), and these questions were not included in the data analysis. In Phase 3, after the experiment was completed, the participant was asked to answer some open-ended questions. The questions are shown in Table 2.

Table 2. Examples of open questions.

Q1: Do you know these calligraphic stamps?

Q2: What impresses you most about calligraphic writing?

Q3: What do you know about Chinese calligraphy culture?

Q4: Have you ever studied Chinese calligraphy? How long have you been studying? 


\section{Results}

The data were first screened and then analyzed, including the treatment of invalid data (censoring) and missing values (mean-replacement method). Two sets of analyses were then performed, one based on global eye movements and the other on local eye movements. Holistic measurements were based on all-gaze components of the entire picture region. They provided a measure of the overall information acquisition and processing difficulty of a region. In contrast, local eye movement measurements reflected the processing time of a much smaller region of the image. By calculating and analyzing the local measures of eye movements, an attempt was made to discover how field cognitive styles differed in the degree of attention paid to different regions and gaze patterns in cursive and running scripts.

\subsection{Overall Analysis}

In this study, the following overall measures were calculated: 1) total fixation counts (TFC, the total number of attempts to gaze at an area); 2) saccade amplitude (SA, the total length of saccade within an area); and 3) saccade counts (SC, the total number of saccade within an area). A 2 (field cognitive style: field independent, field dependent) $\times 2$ (calligraphic style: cursive, running) repeated-measures ANOVA was calculated, and specific eye movement means are shown in Table 3.

Table 3. Means and standard deviations for the cognitive style and the calligraphy style.

\begin{tabular}{ccccc}
\hline & & TFC & SA & SC \\
\hline FD & cursive & $46.42(25.85)$ & $14.27(3.42)$ & $45.88(25.80)$ \\
& running & $61.06(30.09)$ & $11.18(1.88)$ & $60.63(30.09)$ \\
FDI & cursive & $32.87(13.89)$ & $14.52(7.3)$ & $32.46(13.84)$ \\
& running & $51.59(25.65)$ & $12.28(6.01)$ & $51.16(25.50)$ \\
\hline
\end{tabular}

Total fixation counts. ANOVA results showed that the main effect of field cognitive style was significant, $\mathrm{F}(1,76)=4.291, P<0.05, \eta^{2}=0.053$ and the total number of gazes in both cursive and running scripts was greater for field-independent than field-dependent subjects. The main effect of calligraphic style was significant, $\mathrm{F}(1,76)=9.017, p<0.05, \eta^{2}=0.106$. The number of fixation times in the running script was greater than that in the cursive script, and the interaction between field cognitive style and calligraphic style was not significant, $\mathrm{F}(1,76)=0.135, p>0.05, \eta^{2}=0.002$.

Saccade amplitude. The results of ANOVA showed that the main effect of field cognitive style was not significant, $\mathrm{F}(1,76)=0.353, p>0.05, \eta^{2}=0.005$, that of calligraphic style was significant, $\mathrm{F}(1,76)=5.573, p<0.05, \eta^{2}=0.068$ and saccade amplitude was greater in cursive than in running script. The interaction between field cognitive style and calligraphic style was not significant, $\mathrm{F}(1,76)=$ $0.143, p>0.05, \eta^{2}=0.002$. The main effect of calligraphic style was significant, 
with greater saccade amplitude in cursive than in running script, regardless of whether field-independent or field-dependent subjects were tested. The largest saccade amplitude in the cursive script indicated that the information obtained when viewing a cursive work was obtained by viewing more distant locations, whereas a smaller saccade amplitude in the running script indicated that the information obtained was from a location adjacent to the gaze point.

Saccade counts. The results of the ANOVA showed that the main effect of field cognitive style was significant, $\mathrm{F}(1,76)=4.258, p<0.05, \eta^{2}=0.053$, and saccade counts in both cursive and running scripts were greater for the field-independent than the field-dependent subjects. The main effect of calligraphic style was significant, $\mathrm{F}(1,76)=9.10, p<0.05, \eta^{2}=0.107$, with more saccade counts script than in cursive. The interaction between field cognitive style and calligraphic style was not significant $\mathrm{F}(1,76)=0.12, p>0.05, \eta^{2}=0.002$.

\subsection{Local Analysis}

In addition to the global analyses, a set of local analyses were performed in this study, in which smaller regions in columns were considered. The eye movement characteristics of the subjects in these smaller regions were recorded by the average pupil diameter, fixation counts (the number of fixations on a zone of interest), and regression in count measures. Before the results of the eye movement experiments are analyzed statistically, it is necessary to determine the zone of interest of the calligraphic picture, which is the area of interest of the subject's gaze on the stimulus. In order to facilitate direct comparison between cursive and running calligraphy, the study was uniformly divided into three zones of interest: the first (left), the second (middle), and the third (right) zones of interest, depending on the content of the calligraphic picture, from left to right.

Average pupil diameter. In order to investigate whether there are differences in the average pupil diameter between field independence and field dependence in local areas of calligraphic style, a 2 (field cognitive style: field independent, field dependent) $\times 2$ (calligraphic style: cursive, running script) $\times 3$ (area of interest: left, middle, right) ANOVA. The dependent variable was the average pupil diameter. Specific data are shown in Table 4. It was found that the main effect of field cognitive style was significant, $F(1,219)=39.50, p<0.01, \eta^{2}=0.153$, and that the average pupil diameter was greater for the field-independent than for the field-dependent type.

Table 4. Average pupil diameter of cognitive style in different interest areas of calligraphy style.

\begin{tabular}{ccccc}
\hline & & Left & Middle & Right \\
\hline \multirow{2}{*}{ FD } & cursive & $912.07(228.60)$ & $863.04(221.78)$ & $856.58(244.26)$ \\
& running & $896.18(229.86)$ & $892.87(261.26)$ & $850.41(247.05)$ \\
& cursive & $702.08(297.11)$ & $638.09(291.34)$ & $667.71(213.10)$ \\
\hline
\end{tabular}


Fixation counts. Moreover, ANOVA, with field cognitive style, calligraphic style, and area of interest as the independent variables and fixation counts the dependent variables, showed that the main effect of field cognitive style was significant, $\mathrm{F}(1,228)=14.48, p<0.01, \eta^{2}=0.06$, and that fixation counts were higher in the field-independent than in field-dependent type. The main effect of calligraphic style was significant, $\mathrm{F}(1,228)=71.124, p<0.01, \eta^{2}=0.238$, and fixation counts in the first, second, and third areas of interest were higher in running than in cursive script. In addition, the main effect of the area of interest was significant, $\mathrm{F}(2,228)=12.46, p<0.01, \eta^{2}=0.099$, with the second area of interest having the highest fixation counts. Specific data are shown in Table 5.

Table 5. Fixation counts of cognition style in different interest areas of calligraphy style.

\begin{tabular}{ccccc}
\hline & & Left & Middle & Right \\
\hline \multirow{2}{*}{ FD } & cursive & $4.05(2.91)$ & $5.15(2.81)$ & $2.66(2.36)$ \\
& running & $8.44(6.40)$ & $11.26(5.72)$ & $7.51(4.86)$ \\
\multirow{2}{*}{ FDI } & cursive & $3.08(2.16)$ & $3.32(2.35)$ & $0.67(0.67)$ \\
& running & $6.71(5.57)$ & $8.63(6.11)$ & $4.13(3.99)$ \\
\hline
\end{tabular}

Regression in count. With field cognitive style, calligraphy style, and area of interest as the independent variables, ANOVA showed that the main effect of the field cognitive style was significant $\mathrm{F}(1,219)=4.59, p<0.05, \eta^{2}=0.021$, and the regression in counts was higher for the field-independent than the field-dependent type. Calligraphic style and area of interest were found to have significant main effects, $\mathrm{F}(1,219)=18.51, p<0.01, \eta^{2}=0.078$. There was a significant main effect on the area of interest, $\mathrm{F}(2,219)=171.74, p<0.01, \eta^{2}=$ 0.611 ; the interaction between style of calligraphy and area of interest was significant $\mathrm{F}(2,219)=13.11, p<0.01, \eta^{2}=0.107$, and regression in counts was greater in the left and middle parts of the area of interest than in cursive, while regression in counts was greater in cursive calligraphy in the right part of the area of interest. Specific data are shown in Table 6.

Table 6. Regression in count of cognition style in different interest areas of calligraphy style.

\begin{tabular}{ccccc}
\hline & & Left & Middle & Right \\
\hline \multirow{2}{*}{ FD } & cursive & $1.80(0.57)$ & $1.18(0.58)$ & $0.16(0.26)$ \\
& running & $1.85(0.63)$ & $2.05(0.56)$ & 0 \\
\multirow{2}{*}{ FDI } & cursive & $1.60(0.73)$ & $0.71(0.59)$ & $0.42(0.57)$ \\
& running & $1.98(1.04)$ & $1.63(0.92)$ & $0.12(0.38)$ \\
\hline
\end{tabular}

\section{Discussion}

This paper analyzes the eye-movement characteristics of the field cognition style in the aesthetic experience process of two calligraphic styles from a holistic and 
local perspective. Antes (1974) proposed that holistic visual attention can only grasp the general meaning of the stimuli, while local attention is better able to fill in the details and focus on less information. The former is characterized by the integration of information from different locations to identify and extract information from the current location. Its distinctive features are longer saccade times and larger saccade amplitudes. The latter is characterized by exploring for information and extracting detailed information from a specific or nearby location (Liechty, Pieters, \& Wedel, 2003). Therefore, a combination of holistic and local analyses better illustrates the questions of this study in a comprehensive manner. The final findings confirm and extend earlier hypotheses and inferences that field cognitive styles differ in the characteristics of aesthetic experience in calligraphy appreciation and that there are aesthetic preferences in calligraphy styles (Witkin et al., 1954). Specifically, field-independent subjects had richer aesthetic experiences than field-dependent subjects when appreciating calligraphic works, and there were aesthetic preferences between the two work styles, with both field-independent and field-dependent subjects paying more attention to running script works.

In the overall analysis, the aesthetic preferences of field-independent and field-dependent subjects were determined by differences in eye-movement indicators of field cognitive style in the cursive and running script appreciation process. The eye-movement results showed that there were significant differences in field cognitive styles in terms of total fixation counts and saccade counts, with the field-independent subjects having greater fixation counts and saccade amplitude than the field-dependent ones. There were significant differences in calligraphic style in terms of total fixation counts, saccade amplitude, and saccade counts, with line script being significantly larger than cursive and saccade amplitude smaller than cursive. In other words, field-independent and field-dependent subjects with consistent eye movement patterns during cursive and running script appreciation tended to gaze at running calligraphy.

It is believed that the field-independent-field-dependent cognitive approach mostly involves processing of information at the perceptual level (Kozhevnikov, Evans, \& Kosslyn, 2014). In combination with the characteristics of calligraphic works, due to the unique creative style of cursive works, the content of cursive works is not understandable to non-experts, so both field-independent and field-dependent subjects fail to process information at the perceptual level of cursive works and thus fail to have a more aesthetic experience. Contrariwise, since the creative style of running script works is known to the public, the interviewees will give them more attention. According to the information processing stage model of aesthetic experience proposed by Leder the aesthetic experience is divided into five stages: perception, explicit classification, implicit classification, cognitive mastery, and evaluation. Obviously, when appreciating cursive works, the participant fails to acquire information in the first stage, that is, the perception stage, while when appreciating running script works, the participant can ef- 
fectively go through these five stages and finally obtain a certain aesthetic experience. At the same time, from the psychological understanding of aesthetic experience, the higher the degree of understanding of a work of art, the greater the possibility of aesthetic pleasure (Leder, Belke, Oeberst, \& Augustin, 2004).

On the other hand, Martindale's theory of cognitive pleasure postulates that meaning is the primary determinant of aesthetic appreciation, which masks other contents or attributes (Martindale, 1991). Meaning is a function of the following variables: the personal relevance of a painting's components to the viewer, the typicality of its components, and their clarity and naturalness. For both calligraphic styles, the meaning that can be obtained from a running calligraphy work is richer in terms of personal relevance, typicality, or clarity. Furthermore, familiarity with calligraphic works guided subjects' aesthetic processing. This was confirmed by the results of the post-test questionnaire (Hekkert, Snelders, \& Wieringen, 2003). The post-test questionnaire revealed that $57.5 \%$ of the respondents had not seen images of the calligraphy in this experiment before, and $42.5 \%$ of the respondents said they had seen these pictures before. Most of the images were of calligraphic scrolls.

The results of the local analysis showed that there were significant differences in the mean pupil diameter and fixation counts between field cognitive styles, with field-independent styles having larger pupil diameters and fixation counts than field-dependent styles. Yan et al. argued that changes in pupil size can be used as an indicator of the intensity of mental processing. Task-Evoked Pupillary Response (TEPR) is often used to measure cognitive load (Ahern \& Beatty, 1979); the larger the pupil diameter, the more difficult the concurrent processing (Krejtz, Duchowski, Niedzielska, Biele, \& Krejtz, 2018). Moreover, the pupil diameter is also used as an outward indicator of current mood change, with a larger pupil diameter indicating greater mood change. In addition, fixation oculomotor indicators reflected differences in cognitive processing, mental effort, and attentional allocation to visual material, with higher fixation indicators generally implying deeper cognitive processing, greater mental effort, and more complex attentional allocation, while lower fixation indicators corresponded to shallower cognitive processing, less mental effort, and simpler attentional allocation. Therefore, in the appreciation process of local areas in cursive and running scripts, the field cognition style changes according to the different areas of the calligraphic content. In particular, the interaction between the calligraphic style and the area of interest in the number of revisits provides evidence for this. In summary, from the perspective of local analysis, it was shown that there are certain aesthetic differences between field independence and field dependence in the calligraphic aesthetic process. It is noteworthy that there was an interaction between calligraphic style and area of interest in regression in counts. According to the measure of regression in counts, the left part of the screen was more interesting during the appreciation of cursive scripts, while the middle part was more interesting during the appreciation of running scripts. This may be due to 
the fact that the middle part is in the middle of the screen, which attracts more attention, and therefore information processing in this part is more complex (Yarbus, 1967).

Future research can explore the aesthetic characteristics of cognitive styles in the calligraphy aesthetic process based on this study. First of all, the researcher can choose other calligraphic styles, such as regular script, official script, seal script, and so on, as the research material. Different calligraphic styles have their own uniqueness, so it is worthwhile exploring their aesthetic characteristics. Second, the more practical the development of research techniques for visual results visualization, the more beneficial the combination and application of indicators is to the development of the research process and the analysis of results. Therefore, future research on ERP can use a combination of eye movement technologies, and further studies can be conducted on the brain regions activated by characteristics of calligraphy, parts of the aesthetic process, and the corresponding characteristics of eye movement.

\section{Conclusion}

In conclusion, this study analyzed the characteristics of field cognitive style in calligraphy using the eye movement technique and analyzed the aesthetic experience in terms of cognition and emotion. The results showed that field cognitive style has different characteristics in the aesthetic experience in calligraphy appreciation and that there are aesthetic preferences in calligraphy style.

\section{Conflicts of Interest}

The authors declare no conflicts of interest regarding the publication of this paper.

\section{References}

Ahern, S., \& Beatty, J. (1979). During Information Processing Vary with Scholastic Aptitude Test Scores. Science, 205, 1289-1292. https://doi.org/10.1126/science.472746

Antes, J. R. (1974). The Time Course of Picture Viewing. Journal of Experimental Psychology, 103, 62-70. https://doi.org/10.1037/h0036799

Augustin, D., \& Leder, H. (2006). Art Expertise: A Study of Concepts and Conceptual Spaces. Psychology Science, 48, 135.

Boselie, F., \& Leeuwenberg, E. (1985). Birkhoff Revisited: Beauty as a Function of Effect and Means. The American Journal of Psychology, 98, 1-39. https://doi.org/10.2307/1422765

Bradley, M. M., Miccoli, L., Escrig, M. A., \& Lang, P. J. (2010). The Pupil as a Measure of Emotional Arousal and Autonomic Activation. Psychophysiology, 45, 602-607. https://doi.org/10.1111/j.1469-8986.2008.00654.x

Buswell, G. T. (1935). How People Look at Pictures: A Study of the Psychology and Perception in Art. Chicago, IL: University of Chicago Press.

Chu, K. Y., Huang, C. Y., \& Ouyang, W. C. (2018). Does Chinese Calligraphy Therapy Reduce Neuropsychiatric Symptoms: A Systematic Review and Meta-Analysis. BMC 
Psychiatry, 18, 62. https://doi.org/10.1186/s12888-018-1611-4

Cupchik, G. C., \& Gebotys, R. J. (1990). Interest and Pleasure as Dimensions of Aesthetic Response. Empirical Studies of the Arts, 8, 1-14. https://doi.org/10.2190/L789-TPPY-BD2Q-T7TW

Cupchik, G. C., Vartanian, O., Crawley, A., \& Mikulis, D. J. (2009). Viewing Artworks: Contributions of Cognitive Control and Perceptual Facilitation to Aesthetic Experience. Brain and Cognition, 70, 84-91.

https://doi.org/10.1016/j.bandc.2009.01.003

Dong, W., Ying, Q., Yang, Y., Tang, S., Zhan, Z., Liu, B., \& Meng, L. (2019). Using Eye Tracking to Explore the Impacts of Geography Courses on Map-Based Spatial Ability. Sustainability, 11, 76. https://doi.org/10.3390/su11010076

Guisande, M. A., Páramo, M. F., Tinajero, C., \& Almeida, L. S. (2007). Field Dependence-Independence (FDI) Cognitive Style: An Analysis of Attentional Functioning. Psicothema, 19, 572-577.

Hekkert, P., \& Van Wieringen, P. C. (1996). The Impact of Level of Expertise on the Evaluation of Original and Altered Versions of Post-Impressionistic Paintings. Acta Psychologica, 94, 117-131. https://doi.org/10.1016/0001-6918(95)00055-0

Hekkert, P., Snelders, D., \& Wieringen, P. C. W. V. (2003). "Most Advanced, Yet Acceptable": Typicality and Novelty as Joint Predictors of Aesthetic Preference in Industrial Design. British Journal of Psychology, 94, 111-124. https://doi.org/10.1348/000712603762842147

Kao, H. S. R. (2010). Calligraphy Therapy: A Complementary Approach to Psychotherapy. Asia Pacific Journal of Counselling \& Psychotherapy, 1, 55-66. https://doi.org/10.1080/21507680903570334

Kao, H. S., Zhu, L., Chao, A. A., Chen, H. Y., Liu, I. C., \& Zhang, M. (2014). Calligraphy and Meditation for Stress Reduction: An Experimental Comparison. Psychology Research and Behavior Management, 7, 47-52. https://doi.org/10.2147/PRBM.S55743

Kapoula, Z., Daunys, G., Herbez, O., \& Yang, Q. (2009). Effect of Title on Eye-Movement Exploration of Cubist Paintings by Fernand Léger. Perception, 38, 479-491. https://doi.org/10.1068/p6080

Kozhevnikov, M., Evans, C., \& Kosslyn, S. M. (2014). Cognitive Style as Environmentally Sensitive Individual Differences in Cognition: A Modern Synthesis and Applications in Education, Business, and Management. Psychological Science in the Public Interest, 15, 3-33. https://doi.org/10.1177/1529100614525555

Krejtz, K., Duchowski, A. T., Niedzielska, A., Biele, C., \& Krejtz, I. (2018). Eye Tracking Cognitive Load Using Pupil Diameter and Microsaccades with Fixed Gaze. PloS one, 13, e0203629. https://doi.org/10.1371/journal.pone.0203629

Leder, H., Belke, B., Oeberst, A., \& Augustin, D. (2004). A Model of Aesthetic Appreciation and Aesthetic Judgments. British Journal of Psychology, 95, 489-508. https://doi.org/10.1348/0007126042369811

Liechty, J., Pieters, R., \& Wedel, M. (2003). Global and Local Covert Visual Attention: Evidence from a Bayesian Hidden Markov Model. Psychometrika, 68, 519-541. https://doi.org/10.1007/BF02295608

Locher, P. (2006). The Usefulness of Eye Movement Recordings to Subject an Aesthetic Episode with Visual Art to Empirical Scrutiny. Psychology Science, 48, 106.

Martindale, C. (1991). Cognitive Psychology: A Neural-Network Approach. Thomson Brooks/Cole Publishing.

Messick, S. (1984). The Nature of Cognitive Styles: Problems and Promise in Educational 
Practice. Educational Psychologist, 19, 59-74. https://doi.org/10.1080/00461528409529283

Nodine, C., \& Krupinski, E. (2003). How Do Viewers Look at Artworks? Bulletin of Psychology and the Arts, 4, 65-68. https://doi.org/10.1037/e514602010-005

Riding, R., \& Cheema, I. (1991). Cognitive Styles: An Overview and Integration. Educational Psychology, 11, 193-215. https://doi.org/10.1080/0144341910110301

Rizzolatti, G., Riggio, L., Dascola, I., \& Umilta, C. (1987). Reorienting Attention across the Horizontal and Vertical Meridians: Evidence in Favor of a Premotor Theory of Attention. Neuropsychologia, 25, 31-40. https://doi.org/10.1016/0028-3932(87)90041-8

Tennant, M. (1988). Psychology and Adult Learning. London: Routledge.

Vogt, S., \& Magnussen, S. (2007). Expertise in Pictorial Perception: Eye-Movement Patterns and Visual Memory in Artists and Laymen. Perception, 36, 91-100. https://doi.org/10.1068/p5262

Winston, A. S., \& Cupchik, G. C. (1992). The Evaluation of High Art and Popular Art by Naive and Experienced Viewers. Visual Arts Research, 18, 1-14. https://www.jstor.org/stable/20715763

Witkin, H. A., Goodenough, D. R., \& Oltman, P. K. (1979). Psychological Differentiation: Current Status. Journal of Personality and Social Psychology, 37, 1127-1145. https://doi.org/10.1037/0022-3514.37.7.1127

Witkin, H. A., Lewis, H. B., Hertzman, M., Machover, K., Meissner, P. B., \& Wapner, S. (1954). Personality through Perception: An Experimental and Clinical Study. Quarterly Review of Biology, 53, 181-184.

Yarbus, A. L. (1967). Eye Movements during Perception of Complex Objects. In Eye Movements and Vision (pp. 171-211). Boston, MA: Springer.

https://doi.org/10.1007/978-1-4899-5379-7 8

Yu, J., \& Peng, Q. (2005). Realistic Synthesis of Cao Shu of Chinese Calligraphy. Computers \& Graphics, 29, 145-153. https://doi.org/10.1016/j.cag.2004.11.013

Zhang, L. F. (2004). Field-Dependence/Independence: Cognitive Style or Perceptual Ability?-Validating against Thinking Styles and Academic Achievement. Personality and Individual Differences, 37, 1295-1311. https://doi.org/10.1016/j.paid.2003.12.015

Zhuang, Y., Lu, W., \& Wu, J. (2009). Latent Style Model: Discovering Writing Styles for Calligraphy Works. Journal of Visual Communication and Image Representation, 20, 84-96. https://doi.org/10.1016/j.jvcir.2008.11.007 